\title{
Treatment of Spontaneous Pneumothorax by using the Video Assisted Thoracoscopic Surgery and Mini Invasive Thoracoscopic Surgery
}

\author{
Kwang-Hak Ju, Hyon-Jong Kim*, Song-Ho Kim, Yong-Jin Liu
}

Kwang-Hak Ju, Hyon-Jong Kim*, Song-Ho Kim, Yong-Jin Liu

Department of Thoracic Surgery, Pyongyang Medical College, Kim II Sung University, Democratic People's Republic of Korea.

\section{Correspondence}

\section{Mr. Hyon-Jong Kim}

Department of Thoracic Surgery, Pyongyang Medical College, Kim II Sung University, Democratic People's Republic of Korea.

Phone: +850 023670598

Email: pmed8@ryongnamsan.edu.kp History

- Submission Date: 25-12-2018;

- Review completed: 23-01-2019;

- Accepted Date: 10-02-2019.

\section{DOI : 10.5530/ijcep.2018.5.4.24}

\section{Copyright}

(C) 2018 Phcog.Net. This is an openaccess article distributed under the terms of the Creative Commons Attribution 4.0 International license.

\begin{abstract}
Background and Aim: The temporizing treatment for spontaneous pneumothorax had been established methodically, but thoracoscopic surgical methods has been not yet. The objective of the study is to show the treatment effect of intra- and post-operation by using rational thoracoscopic surgery for the patients with spontaneous pneumothorax. Methods: We had performed Video Assisted Thoracoscopic Surgery (VATS) and Mini Invasive Thoracoscopic Surgery (MITS) for 322 patients with spontaneous pneumothorax and established rational surgery approach between January 2011 and December 2017. And then we evaluated the outcome of surgery compared to common thoracotomy. Results: Compared to common thoracotomy, VATS was an operation with large economic efficacy because of light operation associated injury, fast recovery and the ability to decrease temporary disability rate. And also it saved different drugs and provided Quality of life (OOL) because of low operative cicatrix. Conclusion: VATS not only gave light operative burden to patients but also decreased surgeons' mental and physical fatigue. In the MITS automatic suture tool with expensive cost and limited indications was not used and the range of indications were wide and operating time was shortened because of procedure under direct visual field associated with thoracoscopy.

Key words: Spontaneous pneumothorax, Mini invasive thoracoscopic surgery, Video assisted thoracoscopic surgery.
\end{abstract}

\section{INTRODUCTION}

Spontaneous pneumothorax is a disease that air in the lung gets into the pleural cavity because of rupture of bulla and bleb right under pulmonary pleura and then pulmonary parenchyma has collapsed. Emphysematous cyst $<1 \mathrm{~cm}$ in diameter on the surface of lung is called bleb, the one $>1 \mathrm{~cm}$ in diameter in the pulmonary parenchyma is called bulla and it can be developed in any site as well as the apex of lung. In this case, internal pressure of sub-pleural cyst increases and its' wall extends extremely because of inspiratory valve function if bronchioles have fibrosis, bend, constriction and etc. ${ }^{[1]}$ If then it is ruptured at the end, it causes spontaneous pneumothorax. The treatment of spontaneous pneumothorax up to date consists of internal and surgical treatment. The internal treatment includes relaxation, paracentesis, insertion of drain into pleural cavity and administration of pleural adhesion material, while these are not treatment based on evidence, so it can't prevent recurrence. ${ }^{[2]}$ Nowadays, it is one of trends all over the world that thoracoscopic surgery, minimally invasive surgery, is performed even if incipient pneumothorax.

Thoracoscopic surgery can be applied to the patients with cardio-pulmonary failure without relation to age and it shortens postoperative hospitalization days and has no recurrence and complications. ${ }^{[3]}$

The indication of thoracoscopic surgery have been extended remarkably by the introduction of video system to endoscopic surgery, the application of pulmonary ventilation anesthesia and development of different devices for endoscopic surgery.

MITS (Minimally Invasive Thoracoscopic surgery) is mainly similar to VATS while $4-6 \mathrm{~cm}$ minimally thoracotomy is performed in the site for the insertion of automatic suture tool and then ruptured pulmonary part is sutured by pneumonorrhaphy with atraumatic suture (prolen 3-0). Recently not only VATS but also MITS is being performed extensively while MITS is superior to VATS. ${ }^{[4]}$ Because MITS doesn't use automatic suture tool and it extends the range of indication and it shortens the operating time by performing operation under direct visual field associated with thoracoscopy compared to the high usage of automatic suture tool and limited indication including no pleural adhesion. Thus, if it is difficult to operate with VATS, for example intra-operative hemorrhage, adhesion, MITS is performed. ${ }^{[5]}$ Per-

Cite this article: Kwang-Hak J, Hyon-Jong K, Song-Ho K, Yong-Jin L. Treatment of Spontaneous Pneumothorax by using the Video Assisted Thoracoscopic Surgery and Mini Invasive Thoracoscopic Surgery. Int J Clin Exp Physiol. 2018;5(4):208-10. 
forming thoracoscopic surgery, there is no recurrence and complications such as post-operative empyema caused by thoracotomy.

Under the consideration of such situations, we've conducted research work to show the treatment effect of intra- and post-operation by using the rational thoracoscopic surgery for the patients with spontaneous pneumothorax.

\section{MATERIALS AND METHODS}

We subjected 322 patients (male 301, female 21, age: 16-79) with spontaneous pneumothorax who were hospitalized to thoracic surgery department of Pyongyang medical college hospital of Kim Il Sung University from January 2011 to December 2017. We obtained informed consents from all patients in accordance with national medical rules of DPRK. All procedures performed in our studies involving patients were in accordance with the ethical standards of the national medical committee of DPRK.

We performed Thoracoscopy Surgery (TS) in following cases: there're no sign of expanding the collapsed lung for 3-5 days after insertion of drain into pleural cavity and it developed more than 2 times. Patients underwent general anesthesia using a left-sided double lumen endotracheal tube and were placed in supine position, then lateralized to non-lesion side. An incision was made from $5^{\text {th }}$ intercostal space at the middle axillary line. A 15-mm trocar for thoracoscopy was inserted and a 10-mm camera were introduced using this port. Another incision was made from $4^{\text {th }}$ intercostal space at the anterior axillary line for lung forceps. We used to find out the air leakage region undergoing water pressure test, while made an incision at chest wall near this region and auto-stapler was introduced using this port. In some patients, we resected $4 \sim 6 \mathrm{~cm}$ in chest wall corresponding to this port and performed MITS, while ruptured pulmonary part was sutured by pneumonorhaphy with atraumatic suture (prolen 3-0). When air leakage wasn't identified any more, the water in pleural cavity were drawn by suction, a chest tube was inserted from $2^{\text {nd }}$ intercostal space at the middle clavicle line and another one from $8^{\text {th }}$ intercostal space at the posterior axillary line. After that, all incisions sutured respectably. All patients received antibiotics and analgesics were ordered for postoperative pain. Patients were evaluated for several parameter, which was defined according to developing site, composition of cyst, incision length, average time needed for operation, hemorrhage, antibiotics and so on compared TS to thoracotomy.

\section{RESULTS}

As you can see in Table 1, there is no difference according to developing site of spontaneous pneumothorax in left and right site.

As you can see in Table 2, major part of emphysematic cyst developed in superior lobe including pulmonary apex.

As you can see in Table 3, compared to thoracotomy, thoracoscopic surgery, not only provided high value of beauty, but also shortened operating time and the amount of hemorrhage was little.

As you can see in Table 4, compared to thoracotomy, after operation, not only analgesics and antibiotics were a little used absolutely, but also the treatment period and recovery duration were decreased in the thoracoscopic surgery.

\section{DISCUSSION}

Spontaneous pneumothorax is a disease that air in the lung get into the pleural cavity because of rupture of emphysematous cyst right under pulmonary pleura and then pulmonary parenchyma has collapsed. Bleb, emphysematous cyst $<1 \mathrm{~cm}$ in diameter on the surface of lung, occurred by breakdown the alveolar while were cut subpleural connective tissue and was the accumulation of air within the subpleural tissue, being in
Table 1: Developing site of spontaneous pneumothorax ( $n=322)$.

\begin{tabular}{|cccc|} 
& Right & Left & Bilateral \\
\hline $\mathrm{N}(\%)$ & $164(50.9)$ & $156(48.5)$ & $2(0.6)$ \\
\hline
\end{tabular}

Table 2: Composition of emphysematic cyst ( $n=322)$.

\begin{tabular}{cccc} 
& Apex and superior & Middle & Inferior \\
$\mathrm{N}(\%)$ & $308(95.7)$ & $3(0.9)$ & $11(3.4)$ \\
\hline
\end{tabular}

Table 3: Composition of treatment during operation (mean value, $n=348)$.

\begin{tabular}{cccc|} 
& Incision length & $\begin{array}{c}\text { Operating } \\
\text { time }(\mathrm{m})\end{array}$ & $\begin{array}{c}\text { Amount of } \\
\text { hemorrhage } \\
(\mathrm{mL})\end{array}$ \\
\hline VATS $(n=11)$ & $1 \mathrm{~cm} \times 3.3$ & 78.2 & 5.2 \\
MITS $(n=311)$ & $5.7 \mathrm{~cm}$ & 48.6 & 30.7 \\
$\begin{array}{c}\text { Thoracotomy } \\
(\mathrm{n}=26)\end{array}$ & $23.6 \mathrm{~cm}$ & 92.4 & 240.6 \\
\hline
\end{tabular}

Table 4: Treatment composition of post-operation (mean value, $n=348$ ).

\begin{tabular}{|c|c|c|c|c|c|}
\hline & 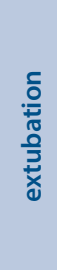 & $\begin{array}{l}\frac{\tilde{v}}{\tilde{y}} \\
\frac{\tilde{\sigma}}{\widetilde{N}} \\
\frac{0}{\sigma}\end{array}$ & $\frac{\tilde{U}}{\frac{\tilde{U}}{0}}$ & 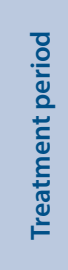 & 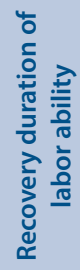 \\
\hline $\operatorname{VATS}(n=11)$ & 2.1 & 1.6 & 2.8 & 5.8 & 10 \\
\hline $\operatorname{MITS}(n=311)$ & 2.3 & 2.1 & 3.2 & 7.2 & 13 \\
\hline Thoracotomy $(n=26)$ & 3.6 & 4.8 & 7.7 & 15.6 & 45 \\
\hline
\end{tabular}

shape of small long balloon. The pathogenesis for bleb may be related to bronchiolar constriction caused by non-specific and specific inflammation, sometimes can be found some pleural adhesion and inflammatory region nearby these area. It can be developed in any site as well as the apex of lung. In this case, internal pressure of subpleural cyst increases and its' wall extends extremely because of inspiratory valve function if bronchioles have fibrosis, bend, constriction and etc. ${ }^{[1]}$ If then it is ruptured at the end, it causes spontaneous pneumothorax.

Bleb is developed at lung apex part very easily, because the internal pressure in there is being higher compared with the basis part obviously. It also may be occurred in tall and thin lung because of ischemia caused by growing rapidly. The rupture of bleb and bulla is to become the main cause of spontaneous pneumothorax, but the rising of air-permeability in cystic wall can be due to spontaneous pneumothorax without rupture and atrophy of cyst. Besides, other causes and risk factors for the development of pneumothorax include empysema, tuberculosis, asthma, pertussis, pneumonia, silicosis, pulmonary fibrosis, pulmonary infarction, bronchitis, bronchiectasis, mediastinal tumor, lung tumor and etc.

The incidence of spontaneous pneumothorax relates to gender (male), growth (height) and air pollution, either, while it's much higher in sever air pollution area. The exercising burden including cough, hiking, golf and etc, can be the cause of spontaneous pneumothorax, but our clinical practice shows that it's developed in many cases without especial factors, being higher in 15-35 years and 10:1 (male: female) for the rate of gender 
composition. The almost of emphysematic cyst developed in superior lobe including pulmonary apex.

The treatment of spontaneous pneumothorax up to date consists of internal and surgical treatment, which have the good and fault in the indication, being argument for different assertion. The internal treatment includes relaxation, paracentesis, insertion of drain into pleural cavity and administration of pleural adhesion material, which being ease the burden associated treatment for patients to surgery, but these are not treatment based on evidence, so it can't prevent recurrence, being longer for treatment duration, having a fault with the avoid of excess-exercise.

Thoracotomy, surgical therapy, is one method by performing operation to suture the ruptured pulmonary part under direct visual field, became most effective operation therapy without the recurrence before. However, because of patients' terror to operation, large injury associated operation, cosmetic problem and so on, it is recently not being wide treatment in the world.

Developing the modern science and technology, TS, light injury and cosmetic therapy, is widely being introduced in clinical practice and lay out new stage in treatment of spontaneous pneumothorax.

TS can classify into VATS (Video Assisted Thoracoscopic Surgery) and MITS (Minimally Invasive Thoracoscopic Surgery), it's having superiority such as short incision length, smaller hemorrhage, short operating time, decreasing the amount of analgesia and antibiotics, short recovery compared to thoracotomy and it can also provide the QOL (Quality of Life).

Patients underwent general anesthesia using a left-sided double lumen endotracheal tube, then lateralized to non-lesion side. 10 -mm camera were introduced from $5^{\text {th }}$ intercostal space at the middle axillary line. Another incision was made from $4^{\text {th }}$ intercostal space at the anterior axillary line for lung forceps. We used to find out the air leakage region undergoing water pressure test, while made an incision at chest wall near this region and auto-stapler was introduced using this port. In some patients, we resected $4-6 \mathrm{~cm}$ in chest wall corresponding to this port and performed MITS, while ruptured pulmonary part was sutured by pneumonorhaphy with atraumatic suture (prolen 3-0). When air leakage wasn't identified any more, a chest tube was inserted from $2^{\text {nd }}$ intercostal space at the middle clavicle line and another one from $8^{\text {th }}$ intercostal space at the posterior axillary line. After that, all incisions sutured respectably.

Nowadays, it is one of trends all over the world that thoracoscopic surgery, minimally invasive surgery and not recurrence, is performed even if incipient pneumothorax.

\section{CONCLUSION}

Compared to common thoracotomy, VATS was an operation with large economic efficacy because of light operation associated injury, fast recovery and the ability to decrease temporary disability rate. And also it saved different drugs and provided QOL (Quality of Life) because of low operative cicatrix. VATS not only gave light operative burden to patients but also decreased surgeons' mental and physical fatigue. In the MITS automatic suture tool with expensive cost and limited indications was not used and the range of indications were wide and operating time was shortened because of procedure under direct visual field associated with thoracoscopy.

\section{Compliance with Ethical Standards}

Before saying Ethical Standards, we will describe about our country. Our country is a socialist society, the patient can receive free charge of treatment and the studies for medical sciences are also supplied from our government. So we don't feel any needs of fund and any conflicts of interest.

In addition, all authors including in this study have no conflicts of interest.

\section{CONFLICT OF INTEREST}

None.

\section{ABBREVIATIONS}

VATS: Video aasisted Thoracoscopic Surgery; MITS: Mini Invasive Thoracoscopic Surgery.

\section{SUMMARY}

The temporizing treatment for spontaneous pneumothorax had been established methodically, but thoracoscopic surgical methods has been not yet. The objective of the study is to show the treatment effect of intra- and post-operation by using rational thoracoscopic surgery for the patients with spontaneous pneumothorax. We had performed Video Assisted Thoracoscopic Surgery (VATS) and Mini Invasive Thoracoscopic Surgery (MITS) for 322 patients with spontaneous pneumothorax and established rational surgery approach between January 2011 and December 2017. And then we evaluated the outcome of surgery compared to common thoracotomy. Compared to common thoracotomy, VATS was an operation with large economic efficacy because of light operation associated injury, fast recovery and the ability to decrease temporary disability rate. And also it saved different drugs and provided Quality of Life (QOL) because of low operative cicatrix. VATS not only gave light operative burden to patients but also decreased surgeons' mental and physical fatigue. In the MITS automatic suture tool with expensive cost and limited indications was not used and the range of indications were wide and operating time was shortened because of procedure under direct visual field associated with thoracoscopy.

\section{REFERENCES}

1. Sahn SA, Heffner JE. Spontaneous pneumothorax. N Engl J Med 2000;342(12):868.

2. Noppen M, DeKeukeleire T. Pneumothorax. Respiration. 2008;76(2):121.

3. Guo Y, et al. Factors related to recurrence of spontaneous pneumothorax. Respirology. 2005;10(3):378.

4. Baumann $\mathrm{MH}$, et al. Management of spontaneous pneumothorax: an American College of Chest Physicians Delphi consensus statement. Chest. $2001 ; 119(2): 590$.

5. Tschopp JM, et al. Management of spontaneous pneumothorax: state of the art. Eur Respir J. 2006;28(3):637.

Cite this article: Kwang-Hak J, Hyon-Jong K, Song-Ho K, Yong-Jin L. Treatment of Spontaneous Pneumothorax by using the Video Assisted Thoracoscopic Surgery and Mini Invasive Thoracoscopic Surgery. Int J Clin Exp Physiol. 2018;5(4):208-10. 